\title{
Intestinal-Type Adenocarcinoma
}

National Cancer Institute

\section{Source}

National Cancer Institute. Intestinal-Type Adenocarcinoma. NCI Thesaurus. Code C4126.

An adenocarcinoma arising from epithelium which has undergone intestinal metaplasia.

Representative examples include gastric, gallbladder, and ampulla of Vater intestinal type adenocarcinomas. 